\title{
Describing global pediatric RSV disease at intensive care units in GAVI-eligible countries using molecular point-of-care diagnostics: the RSV GOLD-III study protocol
}

Yvette N. Löwensteyn ${ }^{1}$, Natalie I. Mazur ${ }^{1}$, Harish Nair ${ }^{2,3}$, Joukje E. Willemsen ${ }^{1}$, Ghislaine van Thiel ${ }^{4}$ and Louis Bont ${ }^{1,3^{*}}$ (1) on behalf of the RSV GOLD III-ICU Network study group

\begin{abstract}
Background: Respiratory syncytial virus (RSV) infection is an important cause of hospitalization and death in young children. The majority of deaths (99\%) occur in low- and lower-middle-income countries (LMICs). Vaccines against RSV infection are underway. To obtain access to RSV interventions, LMICs depend on support from Gavi, the Vaccine Alliance. To identify future vaccine target populations, information on children with severe RSV infection is required. However, there is a lack of individual patient-level clinical data on instances of life-threatening RSV infection in LMICS. The RSV GOLD III—ICU Network study aims to describe clinical, demographic and socioeconomic characteristics of children with life-threatening RSV infection in Gavi-eligible countries.
\end{abstract}

Methods: The RSV GOLD-III-ICU Network study is an international, prospective, observational multicenter study and will be conducted in 10 Gavi-eligible countries at pediatric intensive care units and high-dependency units (PICUs/HDUs) during local viral respiratory seasons for 2 years. Children younger than 2 years of age with respiratory symptoms fulfilling the World Health Organization (WHO) "extended severe acute respiratory infection (SARI)" case definition will be tested for RSV using a molecular point-of-care (POC) diagnostic device. Patient characteristics will be collected through a questionnaire. Mortality rates of children admitted to the PICU and/or HDU will be calculated.

Discussion: This multicenter descriptive study will provide a better understanding of the characteristics and mortality rates of children younger than 2 years with RSV infection admitted to the PICU/HDU in LMICs. These results will contribute to knowledge on global disease burden and awareness of RSV and will directly guide decision makers in their efforts to implement future RSV prevention strategies.

Trial registration number: NL9519, May 27, 2021

Keywords: Respiratory syncytial virus, Children, Pediatric intensive care unit, Study design, Lower-middle-income countries, Burden, Awareness

*Correspondence: I.bont@umcutrecht.nl

${ }^{1}$ Division of Infectious Diseases, Department of Paediatrics, University Medical Centre Utrecht, Utrecht, The Netherlands

Full list of author information is available at the end of the article

\section{Background}

Respiratory syncytial virus (RSV) infection is an important cause of hospitalization and mortality due to lower respiratory tract infection (LRTI) in children under 5 years of age worldwide [1]. Annual RSV-related hospital admissions and in-hospital deaths in this age group 
have been estimated to be 3.2 million and 59,600, respectively, while overall annual RSV-related mortality including community deaths could be as high as 118,200 [1]. The majority of deaths (99\%) occur in low- and lowermiddle-income countries (LMICs) due to lack of access to healthcare and poor quality of care in health facilities [1], and children under 2 years of age are disproportionally affected $[2,3]$. As the Haemophilus influenza type $\mathrm{b}$ and pneumococcal conjugate vaccines are introduced and scaled up in LMICs, the global burden of child pneumonia attributable to bacterial causes has decreased and the proportional contribution of viral pathogens has increased. RSV now remains one of the major pathogens that needs to be tackled in order to achieve sustainable development goal 3.2-end preventable deaths of newborns and children under 5 years of age by 2030 .

Currently there is no immunization available against RSV, although several vaccine and monoclonal antibody candidates are under clinical development [4]. The most advanced maternal vaccine candidate has completed a phase 3 trial but did not meet the primary endpoint [5]. A new extended half-life monoclonal antibody developed by SanofiPasteur / MedImmune, nirsevimab (previously MEDI8897), has met the primary endpoint of reducing RSV LRTI in healthy infants in a recent phase III trial [6].

Gavi, the Vaccine Alliance (previously: Global Alliance for Vaccines and Immunizations), is an international organization that invests in vaccines to protect children's lives and health in LMICs. Every 5 years, Gavi develops a new vaccine investment strategy (VIS) to prioritize new and under-used vaccines and to make these available to LMICs through the Gavi vaccine support programme. RSV interventions, including both maternal vaccine and monoclonal antibodies, were considered as one of the six prioritized vaccine programmes as part of Gavi VIS for the 2021-2025 funding period [7]. It is anticipated that future RSV vaccines or monoclonal antibodies will be most efficacious in targeting severe disease leading to poor outcome (e.g. oxygen supplementation, ICU admission, and death).

The majority of Gavi-eligible countries have sparse or no individual patient-level data to make decisions on target populations for RSV interventions when these become available in the next $5-10$ years. These data will be important for cost-effectiveness analyses of potential RSV interventions to assist policy makers in making decisions related to resource allocation for RSV interventions [8]. Patient-data will also contribute to local disease awareness. Defining burden in terms of RSV incidence and case-fatality ratios in Gavi-eligible countries has been challenging due to insufficient diagnostic capabilities for RSV surveillance [9]. Interviews with stakeholders revealed that RSV prevention received low priority at national and government level due to lack of information about disease and disease burden, and some respondents suggested that RSV diagnostics would help to improve value proposition [10].

This study aims to obtain individual patient-level data from children who have been admitted with severe RSV infection at the (pediatric) intensive care unit (ICU) or high-dependency unit (HDU) in Gavi-eligible countries through implementation of RSV point-of-care testing to pave the way for future vaccine introduction.

\section{Methods/design}

\section{Study design and study site selection}

The RSV GOLD III study is a prospective, observational, multi-centre study and will be conducted at 11 sites in 10 Gavi-eligible countries. The study was initiated at the first study sites in April, 2021. The total duration of the study is 2 years for each participating study site. To select study sites, we sent out an open invitation for collaboration to researchers and physicians from various LMICs from the existing RSV GOLD network. Before the start of the study, we collected information about potential study sites through email correspondence and teleconferences. The minimum collected information included but was not limited to the location and catchment area of the hospital, the hospital staff to be in charge of performing the study, logistics of the hospital (languages spoken, freezer availability, respiratory seasonality), pediatric ward, pediatric or neonatal ICU, and (pediatric) HDU availability and number of beds, the annual number of respiratory illness-related admissions, and mortality rates. If admission data were not available, estimations were made by the study team based on number of beds and information from local collaborators. We selected study sites based on a high expected number of RSV inclusions, quality of the communication and engagement of local collaborators.

\section{Study sites}

The study will be conducted in the following LMICs: Afghanistan, Cameroon, Ghana, Haiti, Mozambique, Nepal (2 hospitals), Nigeria, Sudan, Tanzania, and The Gambia. The study will also be conducted at 2 sites in the Netherlands to allow for a comparison of patient characteristics with patients from a high-income country (HIC). Table 1 provides the characteristics of the participating LMIC study sites. 
Table 1 Characteristics of the participating RSV GOLD III - ICU Network study sites in low- and lower-middle-income countries

\begin{tabular}{|c|c|c|c|c|c|c|}
\hline City, Country & Hospital & $\begin{array}{l}\text { Number of } \\
\text { PICU Beds }\end{array}$ & $\begin{array}{l}\text { Number of } \\
\text { HDU Beds }\end{array}$ & $\begin{array}{l}\text { Number of } \\
\text { NICU beds }\end{array}$ & $\begin{array}{l}\text { Estimated annual number } \\
\text { of children }<2 \text { years } \\
\text { admitted to PICU / HDU } \\
\text { / NICU with severe acute } \\
\text { respiratory infection }\end{array}$ & Respiratory season \\
\hline Zaria, Nigeria & $\begin{array}{l}\text { Ahmadu Bello University } \\
\text { Teaching Hospital }\end{array}$ & NA & $32^{*}$ & 8 & 155 & April-November \\
\hline Mazar-e-Sharif, Afghanistan & Balkh regional hospital & 35 & NA & 8 & 650 & October-March \\
\hline Banjul, The Gambia & $\begin{array}{l}\text { Edward Francis Small Teach- } \\
\text { ing Hospital }\end{array}$ & NA & 20 & 37 & 100 & October-May \\
\hline Khartoum, Sudan & $\begin{array}{l}\text { Jafar Ibn Auf Specialized } \\
\text { Hospital for Children }\end{array}$ & 8 & 9 & 16 & 100 & December-May \\
\hline Kathmandu, Nepal & Kanti Children's Hospital & 12 & 8 & 16 & 130 & July-March \\
\hline Kathmandu, Nepal & $\begin{array}{l}\text { Tribhuvan University Teach- } \\
\text { ing Hospital }\end{array}$ & 4 & 6 & 8 & 32 & July-March \\
\hline Douala, Cameroon & Laquintinie Hospital Douala & 20 & NA & NA & 180 & $\begin{array}{l}\text { September-January } \\
\text { April-June }\end{array}$ \\
\hline Accra, Ghana & Korle Bu Teaching Hospital & 6 & 18 & $50^{* *}$ & 70 & June-November \\
\hline Maputo, Mozambique & Maputo Central Hospital & 21 & NA & $70^{* *}$ & 85 & March-August \\
\hline Dar es Salaam, Tanzania & Muhimbili National Hospital & 13 & NA & $19^{* *}$ & 270 & December-May \\
\hline Port-au-Prince, Haiti & Saint-Damien Hospital & 10 & NA & $16^{* *}$ & 40 & August-January \\
\hline
\end{tabular}

PICU pediatric intensive care unit, $H D U$ high dependency unit, NICU neonatal intensive care unit, NA not available

*Emergency unit serves as HDU

**No recruitment

\section{Study objectives \\ Primary objective}

To describe the clinical, demographic, and socioeconomic characteristics of RSV-positive children under 2 years of age who have been admitted with suspected RSV infection at ICUs or HDUs in Gavi-eligible countries.

\section{Secondary objectives}

1. To determine proportional RSV-related mortality in children under 2 years of age at participating ICUs or HDUs.

2. To compare clinical, demographic, and socioeconomic characteristics between children with fatal and non-fatal RSV infection.

3. To compare clinical, demographic, and socioeconomic characteristics between children with RSV infection from LMICs and HICs.

4. To describe RSV seasonality in the study locations.

5. To compare clinical, demographic, and socioeconomic characteristics between children with (fatal) RSV infection and children with (fatal) influenza infection.
6. To confirm the point-of-care (POC) RSV test using conventional or real-time RSV PCR.

7. To estimate the sensitivity of the World Health Organization (WHO) "extended severe acute respiratory infection (SARI)" case definition for hospitalbased surveillance for severe RSV infection. [11]

8. To compare the burden of RSV infection and mortality rates between children who do and do not meet the WHO "extended SARI" case definition.

\section{Study participants}

For this study, 2 different groups of children (A and B) are distinguished. For each group, a subject must meet all the eligibility criteria in order to participate (Table 2). Children from group A will be tested for RSV at all study sites and for influenza at 3 study sites (Ghana, Mozambique, Nepal) (Fig. 1):

\section{Group A. Children with suspected RSV disease (all study sites)}

1. Children $<2$ years of age at time of sampling;

2. Who are admitted to an ICU and/or HDU and meet the WHO "extended SARI" case definition; 
Table 2 Eligibility criteria for RSV GOLD III-ICU Network Study

\begin{tabular}{|c|c|}
\hline \multicolumn{2}{|l|}{ Inclusion criteria } \\
\hline Group A & Group B \\
\hline $\begin{array}{l}\text { Age }<2 \text { years at time of sampling; } \\
\text { Admitted to PICU / HDU / NICU; } \\
\text { Meeting WHO “Extended SARI" case definition: } \\
\text { Severe (defined as requiring hospitalization); and } \\
\text { Acute (defined as onset within the last } 10 \text { days); and } \\
\text { Respiratory infection (defined as having cough or shortness of breath) } \\
\text { In infants less than } 6 \text { months, additionally include those who present with: } \\
\text { Apnea (temporary cessation of breathing from any cause); and/or } \\
\text { Sepsis, defined as: } \\
\text { Fever ( } 37.5^{\circ} \mathrm{C} \text { or above) or hypothermia (less than } 35.5^{\circ} \mathrm{C} \text { ); and } \\
\text { Shock (defined as lethargy, fast breathing, cold skin, prolonged capillary } \\
\text { refill or fast weak pulse); and } \\
\text { Seriously ill with no apparent cause } \\
\text { Signed and dated written informed (deferred) consent obtained from the } \\
\text { parent(s)/legal representative(s) of the subject, or in accordance with local } \\
\text { regulations }\end{array}$ & $\begin{array}{l}\text { Age }<2 \text { years at time of sampling; } \\
\text { Admitted to PICU / HDU / NICU; } \\
\text { Signed and dated written informed (deferred) consent obtained from the } \\
\text { parent(s)/legal representative(s) of the subject, or in accordance with local } \\
\text { regulations }\end{array}$ \\
\hline
\end{tabular}

PICU pediatric intensive care unit, HDU high dependency unit, NICU neonatal intensive care unit, WHO world health organization, SARI severe acute respiratory infection

Children who do not meet the WHO "extended SARI" case definition will be tested for RSV at 3 study sites (group B):

\section{Group B. Children who are not suspected to have RSV disease (Ghana, Mozambique, Nepal)}

1. Children $<2$ years of age at time of sampling;

2. Who are admitted to an ICU and/or HDU and do not meet the WHO "extended SARI" case definition;

\section{WHO "extended SARI" RSV surveillance case definition}

In this study, the global case definition developed by the WHO for hospital-based RSV surveillance is used to identify children hospitalized with suspected RSV infection. The RSV surveillance case definition was recently modified based on results from the WHO RSV surveillance pilot which showed that the use of an extended SARI case definition, not requiring fever to identify a suspect case, substantially increased the number of RSV infections detected [3].

In group A, subjects will be tested for RSV when they are admitted to the ICU meeting the WHO "extended SARI" case definition for hospital-based surveillance for severe RSV infection:

- Severe (defined as requiring hospitalization); and

- Acute (defined as onset within the last 10 days); and

- Respiratory infection (defined as having cough or shortness of breath)

In infants less than 6 months, additionally include those who present with:
- Apnea (temporary cessation of breathing from any cause); and/or

- Sepsis, defined as:

- Fever $\left(37.5{ }^{\circ} \mathrm{C}\right.$ or above) or hypothermia (less than $\left.35.5^{\circ} \mathrm{C}\right)$; and

- Shock (defined as lethargy, fast breathing, cold skin, prolonged capillary refill or fast weak pulse); and

- Seriously ill with no apparent cause

In group B subjects not fulfilling the WHO "extended SARI" case definition will also be tested for RSV.

\section{Exclusion criteria}

Neonates younger than 4 days old will not be tested for RSV or influenza due to the high incidence of respiratory symptoms related to intrapartum-related complications or prematurity in this group.

\section{Sample collection}

A nasopharyngeal swab (flocked swab, COPAN, $3 \mathrm{ml}$ universal transport medium (UTM)) will be obtained as soon as possible but no later than $72 \mathrm{~h}$ after admission to the PICU and/or HDU. Samples will be taken by trained healthcare staff.

\section{Point-of-care testing}

The samples will be tested for RSV and influenza using the highly sensitive and specific point-of-care (POC) ID NOW test [12-14]. On-site training for local study staff on performing the POC test will be provided by the RSV GOLD team. If site visits are not possible, the training 


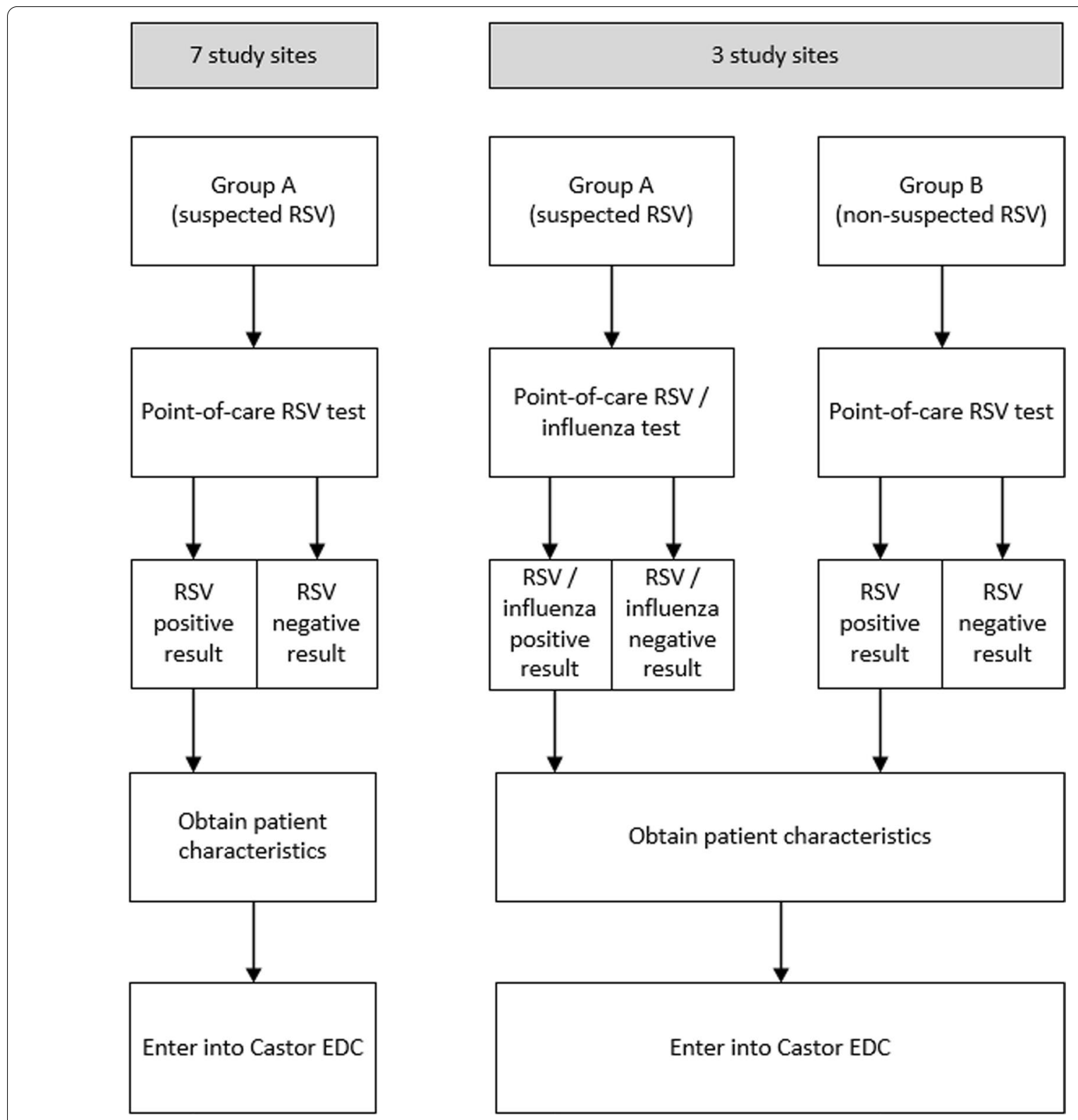

Fig. 1 Schematic diagram of the study workflow. Group A: children < 2 years admitted to the PICU and/or HDU meeting the WHO "extended SARI" case definition (10 study sites). Group B: children $<2$ years admitted to the PICU and/or HDU not meeting the WHO "extended SARI" case definition (3 study sites: Ghana, Mozambique, Nepal)

will be given online. Refresher training will be provided before the start of each new respiratory season or if required by the study sites.

\section{Point-of-care test confirmation}

Although the POC ID NOW RSV test has shown high sensitivity and specificity of $100 \%$ and $97 \%$, respectively, in previous studies [13], polymerase chain reaction (PCR) remains the gold standard for confirmation of a positive or negative test. POC RSV test confirmation is an optional part of the protocol. Samples will preferably be shipped to the University Medical Centre Utrecht (UMCU) laboratory for conventional or real-time PCR testing in order to confirm the POC RSV test and ensure quality of the data. Researchers may decide to perform viral testing and/or sequencing at a local, national or academic reference laboratory accredited to international quality standards with validated conventional or real-time RSV PCR tests when the capacity is available or when shipment of samples to UMCU is not possible. 
Per study site, the following samples will be confirmed:

- All RSV-positive samples.

- A matched (by age and month of admission) number of RSV-negative samples.

\section{Sample storage}

Nasopharyngeal samples will be stored in freezers before shipment. For budget restrictions, influenza-positive samples will not be confirmed through PCR and will not be stored.

\section{RSV sequencing}

RSV sequencing is an optional part of the protocol. Samples sent for confirmation will be stored at the UMCU or study site to allow for sequencing if the sample is RSVpositive. Investigating the molecular heterogeneity of RSV isolates can be important to determine susceptibility or resistance to future RSV monoclonal antibodies or vaccines.

\section{Data collection and data management}

From each study site baseline data will be obtained using questionnaires to evaluate local clinical treatment and management availability and standards. Included patients will be followed up until death or discharge. Participant data will be collected through a case report form and parental questionnaire (Additional file 1: Table S1). These data will be entered by hospital study staff into Castor Electronic Data Capture (EDC) system [15]. Data validation, data analysis and interpretation of the data will be performed by the Utrecht-based study team in collaboration with the site investigators.

\section{Sample size}

RSV

The aim is to include all children $<2$ years of age meeting the case definition admitted to the PICU and/or HDU at each study site each year during the respiratory season. There is no maximum number of patients each study site needs to recruit. We estimated the following number of inclusions for all study sites (Table 3):

\section{Group A}

Based on an estimated number of admissions, we expect to recruit 2800 patients across 10 study sites, 100-400 patients at each study site. Assuming 30\% of patients who fulfill the WHO case definition "extended SARI" will have a positive RSV test, we expect to capture 840 RSV-positive children, 30-120 children at each study
Table 3 Estimated number of RSV-positive patients and RSVrelated deaths

\begin{tabular}{llll}
\hline & $\begin{array}{l}\text { RSV-positive patients } \\
\text { per study site (N, range) }\end{array}$ & $\begin{array}{l}\text { Total RSV- } \\
\text { positive } \\
\text { patients (N) }\end{array}$ & $\begin{array}{l}\text { Total RSV- } \\
\text { related } \\
\text { deaths (N) }\end{array}$ \\
\hline Group A & $30-120$ & 840 & 84 \\
Group B & $10-30$ & 60 & 6 \\
Total & NA & 900 & 90 \\
\hline
\end{tabular}

site. Assuming a mortality rate of $10 \%$ we expect approximately 3-12 RSV-related deaths at each study site, in total $\mathrm{N}=84$ deaths. We consider this number sufficient for descriptive purposes.

If 2800 patients will be recruited and 30\% RSV-positives are observed $(\mathrm{N}=840)$, this produces a two-sided 95\% Clopper-Pearson confidence interval with a width equal to 0.034 , ranging from 28 to $32 \%$. In the smallest estimate, 100 patients will be recruited per site. With an assumed RSV-positive sample proportion of $30 \%$, a sample size of 100 patients produces a two-sided 95\% confidence interval with a width equal to 0.187 , with a lower limit of $21 \%$ and an upper limit of $40 \%$.

In $840 \mathrm{RSV}$-positive patients, an observed proportion of mortality of $10 \%$ will produce a two-sided $95 \%$ confidence interval with a width equal to 0.042 , producing a lower limit of $8.1 \%$ and an upper limit of $12.2 \%$. In the smallest estimate, 100 patients will be recruited per site. With an observed mortality proportion of $10 \%$, a sample size of 100 patients produces a two-sided $95 \%$ confidence interval with a width equal to 0.127 , corresponding to a lower limit of $4.9 \%$ and an upper limit of $17.6 \%$. We consider those estimates sufficiently precise.

\section{Group B}

We expect to recruit 1200 patients in 3 study sites, 200600 patients at each study site. Assuming 5\% of patients will have a positive RSV test, we expect to capture 60 RSV-positive children, 10-30 children at each study site. Assuming a mortality rate of $10 \%$ in children who tested positive for RSV, we expect an additional 1-3 RSVrelated deaths at each study site, in total $\mathrm{N}=6$ deaths.

When the sample proportion is $5 \%$, a sample size of 1200 patients produces a two-sided 95\% confidence interval with a width equal to 0.026 , corresponding to a confidence interval from 3.8 to $6.4 \%$.

In $60 \mathrm{RSV}$-positive patients, an observed proportion of mortality of $10 \%$ will produce a two-sided $95 \%$ confidence interval with a width equal to 0.167 , with a lower limit of $3.8 \%$ and an upper limit of $20.5 \%$. 


\section{Sensitivity WHO case definition}

The sensitivity of the WHO "extended SARI" case definition will be calculated using the total population from the 3 sites that included group A and B for RSV testing. In total, we expect to recruit 800 patients from group A and 1200 patients from group B at 3 study sites, in total 2000 patients. We will not adapt the sample size for this purpose as this is a secondary endpoint of the study.

\section{Influenza (secondary objective)}

Per site we expect to test for influenza in 100-400 (group A) children. Assuming a $5 \%$ positivity rate we expect to capture 42 influenza-positive children, 5-20 children at each study site. Assuming a mortality rate of $10 \%$, we expect approximately 1-2 influenza-related deaths at each study site for group $\mathrm{A}$, in total $\mathrm{N}=4$ deaths.

\section{Statistical analysis}

We will describe characteristics of RSV-positive children. Chi-square tests and nonparametric tests will be used to compare clinical and demographic characteristics between children where appropriate. RSV-positive children from group A and group B will be presented as proportions with $95 \%$ confidence intervals. The estimated case fatality ratio in RSV positive children will be provided with $95 \%$ confidence intervals. We will also report the mortality rate with 95\% confidence intervals, and total RSV-related mortality in group A and B. Subgroup analysis per site will also be performed. We will calculate the sensitivity of the WHO case definition "extended SARI". We will divide the number of RSV-positive children meeting the case definition (group A) by the total number of RSV-positives regardless of whether the case definition was met and express it as a percentage. No formal statistical analysis plan was written before the start of this descriptive study.

\section{Burden of disease}

The estimated number of RSV-related PICU and/or HDU admissions and deaths in children with respiratory infection in the specific country of participating study sites will be quantified. A numerator (the number of POC-confirmed RSV-related admissions and deaths at the PICU and/or HDU) and denominator (population in the hospital catchment area) will be defined. In case the catchment population is not readily available because the facility is not the only one providing in-patient care to the population, it will be estimated based on reviewing hospital administrative datasets and using a hospital admission survey [16]. In the case of limited administrative data or limited resources to perform a hospital admission survey, burden of RSV disease may be described in terms of the proportion of RSV-related PICU and/or HDU admissions (or deaths) among all PICU and/or HDU admissions with LRTI. Other markers of disease burden will also be reported, such as length of stay, duration of oxygen supplementation, etc.

\section{Ethical considerations}

The study will be conducted according to the principles of the Declaration of Helsinki (version 2013), and local law and regulations. Risks and burdens for study subjects are considered minimal. No other safety issues are expected due to the set-up and nature of the study. No Data Safety Monitoring Board will be appointed and no (Severe) Adverse Events will be reported. Written informed consent will be obtained from each patientparticipant by research staff or in accordance with local regulations prior to enrolment in the study.

The intended benefits resulting from this study can be divided into 1) direct benefits and 2) indirect benefits. The primary direct benefit for study participants is timely and proper diagnosis of RSV infection which may result in the prevention of unnecessary or inappropriate use of antibiotics. Secondary direct (patient) benefits consist of:

a) The ability to determine that RSV is not the cause of disease and that an alternative diagnosis should be considered in case of a negative test;

b) The ability to provide parents of RSV-positive children who have been admitted to PICU and/or HDU or who died with information about the cause of or contribution to death.

Indirect (societal) benefits consist of:

a) The ability to provide information on disease burden and target populations to policy makers when a vaccine becomes available;

b) Giving medical staff insights into the incidence of RSV/influenza-related admissions and mortality at their hospital;

c) Identifying RSV and influenza as important causes of PICU and/or HDU admission and death;

d) Capacity building by supplying a reliable POC test to confirm or rule out RSV and influenza as a cause of respiratory infection;

e) Capacity building through involving local site investigators in conducting clinical research;

f) Increasing overall RSV awareness of hospital staff and parents of young children.

For children from HICs, no direct benefits apply, as RSV testing is part of routine care. 
Dual ethical review was performed to ensure that the ethical standards in this study are no less stringent than those applicable in the country of the sponsoring organization. This protocol was therefore submitted for ethics review in The Netherlands as well as to all local (and/or national) research ethics committees.

\section{Discussion}

Although global estimates show a high RSV burden in children from LMICs, individual patient-level data are lacking due to limited availability of RSV testing in these countries. The critical lack of diagnostic capacity hampers the ability to distinguish RSV from other causes of severe respiratory infection in children to justify the need for vaccine introduction in LMICs. Disease awareness is essential to introduce RSV interventions, which will become available in the near future. RSV GOLD is the first global online registry for children younger than 5 years who have died with RSV infection. The first results of the RSV GOLD study have been previously published $[2,17]$. The registry was extended after publication (RSV GOLD II) and data collection is still ongoing. In order to increase real-time data collection from LMICs, funding was obtained to establish a network of PICUs in 10 different Gavi-eligible countries (RSV GOLD III).

The RSV GOLD III-ICU Network study is a novel collaboration between RSV GOLD and 10 study teams from 3 different continents, aiming at collecting individual patient-level data of young children with severe RSV infection through POC RSV testing. Data analyses will provide insights into potential risk factors for fatal RSV infection at the PICU and/or HDU and differences between patients from various income settings.

Some challenges remain for this type of study. First, this study will take place mainly in tertiary level facilities in urban areas where access to healthcare is likely better than in rural areas. Results may therefore not be representative of the whole country. Second, the definition of an ICU and HDU may differ from country to country and even within a single healthcare system. For example, in some countries, the capacity to mechanically ventilate differentiates a PICU bed from a HDU bed, while in other countries, a PICU bed may be defined as a bed within a hospital area with a higher patient: nurse ratio. In 2017, the task force of The World Federation of Societies of Intensive and Critical Care Medicine proposed a global definition and stratified ICUs based on the intensity of care provided [18]. For this study, we included both PICUs and HDUs according to the definition of the participating study sites, where the most severely ill children are usually admitted. To characterize differences between participating PICUs and HDUs, we will collect information on the capacity of care, such as the number of available ventilators and attending healthcare staff. Third, due to budgetary constraints, we are unable to extend RSV testing to the regular wards. We will therefore likely miss a proportion of potential study participants in case PICU and/or HDU beds are occupied and children with severe respiratory infection are admitted to the regular wards instead. We will make an effort in collecting information on the number of refusals to estimate the potential impact of this study limitation. Also, influenza testing is limited to 3 study sites. However, this study will provide insights into the characteristics of hospitalized children with severe RSV infection in LMICs including complete granular age distribution data which can be used for modelling studies on the impact of upcoming maternal vaccines and monoclonal antibodies against RSV. Fourth, for some study sites it may be difficult to calculate a catchment population due to the presence of other PICUs in the area. We will collect information on the number of other available PICUs and will adjust for this in our calculations. Based on preliminary data we estimate that 2-4 study sites will not have sufficient data to estimate the catchment population.

Finally, the SARS-CoV2 pandemic may affect the number of respiratory admissions, thus study results may not be representative of regular respiratory seasons in participating countries. Since the duration of the study is 2 years and most of the recruitment will take place in 2022, we do not expect this to be a major limitation.

In summary, this global prospective multicenter study will provide a better understanding of the characteristics and mortality rates of children younger than 2 years who are admitted to the PICU and/or HDU with severe RSV infection in LMICs. These results will not only contribute to knowledge on global disease burden and awareness of RSV, but will also provide valuable information to healthcare policy makers on the impact of future RSV prevention strategies.

\section{Abbreviations \\ RSV: Respiratory Syncytial Virus; LRTI: Lower respiratory tract infection; LMIC: Low- and lower-middle income country; VIS: Vaccine investment strategy; ICU: Intensive care unit; HDU: High-dependency unit; NICU: Neonatal intensive care unit; HIC: High-income country; WHO: World Health Organization; SARI: Severe acute respiratory infection; UTM: Universal transport medium; POC: Point-of-care; PCR: Polymerase chain reaction; UMCU: University Medical Centre Utrecht; EDC: Electronic data capture.}

\section{Supplementary Information}

The online version contains supplementary material available at https://doi. org/10.1186/s12879-021-06544-3.

Additional file 1. Table S1. Patient variables collected. 


\section{Acknowledgements}

In addition, we thank the following study staff from participating study sites:

Fatima Abdullahi, Hamdalla Adelaiye, Abiola Oloruookoba, Fadlulah Abdurahman, Halima Mudasir, Abdulrahman Zakariya, Nana Aisha Makarfi, Suleiman Bello (Ahmadu Bello University Teaching Hospital), Amie Conteh, Musa Jaiteh, Sheikh Joof, Momodou Lamin Jobarteh, Ansumana Badjie, Mariama A. Jallow, Abdoulie Garba (Edward Francis Small teaching Hospital), Dr. Safaa Medani (Alneelain University), Khalid Enan, Dina N Abdelrahman, Mohamed O Mustafa, Razan A Bashir, Tarteel Hassan, Hassan Madni, Mutaz A Elsir, Awadalkareem Y Awadalkareem, Musab D Hassan, Abdel Rahim M El Hussein (Virology Department Central Laboratory Sudan),

Eugenia Macassa, Stella Langa, Afsha Tahibo, W. Chris Buck (Maputo Central Hospital), Jahit Sacarlal, Samuel Simbine, Alfeu Passanduca (Eduardo Mondlane University Medical School), Prakash Joshi (Kanti Children's Hospital), Rupesh Shrestha (Tribhuvan University Teaching Hospital), Bekolo Cavin Epie, Agnes Eyoh (Laquintinie hospital Douala), Missael In Leger, Jean Peterson Noel Fils, Alexandra Deroncelay, Jamesley Joseph (Saint-Damien Hospital).

We also thank Trisja Boom, Safia Laqqa, and Renate Visser for their contributions to setting up this study. Furthermore, we thank Prachi Vora and Padmini Srikantiah for their scientific advice.

The RSV GOLD III-ICU Network study group authors are (listed in hospital alphabetical order): Ahmadu Bello University Teaching Hospital, Zaria, Nigeria (Maria Ahuoiza Garba, Fatima Jumai Giwa); Balkh regional hospital, Mazar-eSharif, Afghanistan (Mohammad Hafiz Rasooly, Aminullah Shirpoor, Merwais Azizyar); Edward Francis Small Teaching Hospital, Banjul, The Gambia (Lamin Makalo, Ousman Nyan); Jafar Ibn Auf Specialized Hospital for Children, Khartoum, Sudan (Ali Mohamed, Khalid Osman); Kanti Children's Hospital, Kathmandu, Nepal (Ram Hari Chapagain, Krishna Prasad Bista); Tribhuvan University Teaching Hospital, Kathmandu, Nepal (Arun Kumar Sharma, Prabina Shrestha); Korle Bu Teaching Hospital, Accra, Ghana (Bamenla Goka, Kwabena Osman); Noguchi Memorial Institute for Medical Research (Evangeline Obodai); Laquintinie hospital, Douala, Cameroon (Henshaw Mandi, Lucas Esuh Esong, Charlotte Ekoube Eposse); Maputo Central Hospital, Maputo, Mozambique (Valéria Muando) and Eduardo Mondlane University Medical School (Tufária Mussá); Muhimbili National Hospital, Dar es Salaam, Tanzania (Yasser Habresh Said, Aika Abia Shoo); Saint-Damien Hospital, Port-au-Prince, Haiti (Vanessa Jaelle Dor, Jacqueline Gautier, Lynda Abicher).

\section{Authors' contributions}

$L B$ and $Y L$ designed and co-authored the protocol. All site investigators from the RSV GOLD III-ICU Network study group reviewed the protocol and provided feedback. YL and LB drafted the initial manuscript. All authors read, commented on and approved the final manuscript version. All authors read and approved the final manuscript.

\section{Funding}

This study is funded by the Bill \& Melinda Gates Foundation. The funder reviewed the study protocol and provided feedback, but has no role in the study design, data collection, data analysis, data interpretation, writing of the report, or the decision to submit the protocol or future results for publication.

\section{Availability of data and materials}

Not applicable.

\section{Declarations}

\section{Ethics approval and consent to participate}

The study protocol (version 1.0, date 01-08-2020) was reviewed by the Research Ethics Committee of University Medical Centre Utrecht (METC Utrecht), the Netherlands (20-536). A waiver was provided for ethics review of participating Dutch sites (date 29-09-2020). For dual review purposes, the METC Utrecht reviewed the protocol with a focus on ethical conduct of research in low resource settings, using the Declaration of Helsinki and CIOMS guidelines. The study protocol was subsequently reviewed by all site investigators and comments were incorporated into the new version (version 1.2, date 15-11-2020). Ethics approval was obtained by the Institutional Review Boards of participating study sites:Afghanistan: Institutional Review Board, Ministry of Public Health: A.0121.0263;Cameroon: Cameroon Bioethics Initiative Ethics Review and Consultancy Committee: 1156;Ghana: Korle Bu Teaching Hospital Scientific and Technical Committee: KBTH-STC 00056/2021;Haiti:
Comité National de Bioéthique c/o Association Médicale Haitienne: Ref 2021-19;Mozambique: Comité Nacional de Bioética para Saúde: IRB00002657; Ref:344/CNBS/21;Nepal: Ethical Review Board (ERB), Nepal Health Research Council (NHRC): 849/2020; Institutional Review Committee (IRC), Institute of Medicine, Tribhuvan University: 391/077/078; Institutional Review Committee (IRC), Kanti Children's Hospital: 50/2020-021;Nigeria: Health Research Ethics Committee, Ahmadu Bello University Teaching Hospital Shika, Zaria, Nigeria: ABUTHHZ/HREC/H22/2021;Sudan: National Health Research Ethics Review Committee: 7-1-21;Tanzania: Muhimbili National Hospital Institutional Ethics Review Board: MNH/IRB/I/2021/39;The Gambia: Edward Francis Small Teaching Hospital Research \& Ethics Committee: $27^{\text {th }}$ July, 2021.The study has been registered in the Netherlands Trial Register (NL9519) on May 27, 2021.Informed consent will be obtained from the parent(s) or legal representative(s) by research staff prior to enrolment in the study.

\section{Consent for publication}

Not applicable.

\section{Competing interests}

LJB has regular interaction with pharmaceutical and other industrial partners. He has not received personal fees or other personal benefits. UMCU has received major funding (>€100,000 per industrial partner) for investigator-initiated studies from AbbVie, Medlmmune, Janssen, the Bill and Melinda Gates Foundation, Nutricia (Danone) and MeMed Diagnostics. UMCU has received major cash or in kind funding as part of the public private partnership IMIfunded RESCEU project from GSK, Novavax, Janssen, AstraZeneca, Pfizer and Sanofi. UMCU has received major funding by Julius Clinical for participating in the INFORM study sponsored by MedImmune. UMCU has received minor funding for participation in trials by Regeneron and Janssen from 2015 to 2017 (total annual estimate less than $€ 20,000$ ). UMCU received minor funding for consultation and invited lectures by AbbVie, Medlmmune, Ablynx, Bavaria Nordic, MabXience, Novavax, Pfizer, Janssen (total annual estimate less than $€ 20,000)$. LJB is the founding chairman of the ReSViNET Foundation. NIM has regular interaction with pharmaceutical and other industrial partners. She has not received personal fees or other personal benefits. HN reports grants from Innovative Medicines Initiative and National Institute of Health Research; personal fees and grants from WHO and Sanofi; and personal fees from the Bill \& Melinda Gates Foundation, Janssen, Abbvie, and Reviral.GvT is involved in public private partnerships (EU Innovative Medicines Initiative consortia). She has not received personal fees or other personal benefits.

\section{Author details}

${ }^{1}$ Division of Infectious Diseases, Department of Paediatrics, University Medical Centre Utrecht, Utrecht, The Netherlands. ${ }^{2}$ Centre for Global Health, Usher Institute, Edinburgh Medical School, University of Edinburgh, Edinburgh, UK. ${ }^{3}$ Respiratory Syncytial Virus Network (ReSViNET) Foundation, Zeist, The Netherlands. ${ }^{4}$ Julius Center for Health Sciences and Primary Care, Medical Humanities Department, University Medical Center Utrecht, Utrecht, The Netherlands.

Received: 28 June 2021 Accepted: 6 August 2021

Published online: 23 August 2021

\section{References}

1. Shi T, McAllister DA, O'Brien KL, Simoes EAF, Madhi SA, Gessner BD, et al. Global, regional, and national disease burden estimates of acute lower respiratory infections due to respiratory syncytial virus in young children in 2015: a systematic review and modelling study. Lancet. 2017;390(10098):946-58.

2. Scheltema NM, Gentile A, Lucion F, Nokes DJ, Munywoki PK, Madhi SA, et al. Global respiratory syncytial virus-associated mortality in young children (RSV GOLD): a retrospective case series. Lancet Glob Health. 2017;5(10):e984-91.

3. Hirve S, Crawford N, Palekar R, Zhang W, Group WRs. Clinical characteristics, predictors, and performance of case definition-Interim results from the WHO global respiratory syncytial virus surveillance pilot. Influenza Other Respir Viruses. 2019. https://doi.org/10.1111/irv.12688.

4. PATH. RSV Vaccine and mAb Snapshot 2020. Available from: https://www. path.org/resources/rsv-vaccine-and-mab-snapshot/. Accessed 22 Jun 2021. 
5. Madhi SA, Polack FP, Piedra PA, Munoz FM, Trenholme AA, Simoes EAF, et al. Respiratory syncytial virus vaccination during pregnancy and effects in infants. N Engl J Med. 2020;383(5):426-39.

6. Nirsevimab MELODY Phase III trial met primary endpoint of reducing RSV lower respiratory tract infections in healthy infants. https://www.astra zeneca.com/media-centre/press-releases/2021/nirsevimab-phase-iii-trialmet-primary-endpoint.html. Accessed 22 Jun 2021.

7. Gavi, The Vaccine Alliance. https://www.gavi.org. Accessed 22 Jun 2021.

8. Zhang S, Akmar LZ, Bailey F, Rath BA, Alchikh M, Schweiger B, et al. Cost of respiratory syncytial virus-associated acute lower respiratory infection management in young children at the regional and global level: a systematic review and meta-analysis. J Infect Dis. 2020;222:S680-7.

9. Geoghegan S, Erviti A, Caballero MT, Vallone F, Zanone SM, Losada JV et al. Mortality due to respiratory syncytial virus burden and risk factors. Am J Respir Crit Care Med. 2017;195(1):96-103.

10. Gavi, The Vaccine Alliance. Vaccine investment strategy. https://www.gavi. org/our-alliance/strategy/vaccine-investment-strategy. Accessed 22 Jun 2021

11. World Health Organization. RSV surveillance case definitions 2020. https://www.who.int/teams/global-influenza-programme/global-respi ratory-syncytial-virus-surveillance/case-definitions. Accessed 22 Jun 2021.

12. Schnee SV, Pfeil J, Ihling CM, Tabatabai J, Schnitzler P. Performance of the Alere i RSV assay for point-of-care detection of respiratory syncytial virus in children. BMC Infect Dis. 2017;17(1):767.
13. Peters RM, Schnee SV Tabatabai J Schnitzler P Pfeil J. Evaluation of Alere i RSV for rapid detection of respiratory syncytial virus in children hospitalized with acute respiratory tract infection. J Clin Microbiol. 2017;55(4):1032-6.

14. Abbott. ID NOW RSV 2020. https://www.alere.com/en/home/productdetails/id-now-rsv-us.html. Accessed 22 Jun 2021.

15. Castor Electronic Data Capture system 2021. https://www.castoredc.com/ electronic-data-capture-system/. Accessed 22 Jun 2021.

16. World Health Organization. A manual and a supplement for estimating disease burden associated with seasonal influenza 2015. https://apps. who.int/iris/handle/10665/178801. Accessed 22 Jun 2021.

17. Löwensteyn YN, Phijffer E, Simons JVL, Scheltema NM, Mazur NI, Nair $\mathrm{H}$, et al. Respiratory syncytial virus-related death in children with down syndrome: the RSV GOLD Study. Pediatr Infect Dis J. 2020. https://doi.org/ 10.1097/INF.0000000000002666.

18. Marshall JC, Bosco L, Adhikari NK, Connolly B, Diaz JV, Dorman T, et al. What is an intensive care unit? A report of the task force of the World Federation of Societies of Intensive and Critical Care Medicine. J Crit Care. 2017;37:270-6.

\section{Publisher's Note}

Springer Nature remains neutral with regard to jurisdictional claims in published maps and institutional affiliations.
Ready to submit your research? Choose BMC and benefit from:

- fast, convenient online submission

- thorough peer review by experienced researchers in your field

- rapid publication on acceptance

- support for research data, including large and complex data types

- gold Open Access which fosters wider collaboration and increased citations

- maximum visibility for your research: over $100 \mathrm{M}$ website views per year

At BMC, research is always in progress.

Learn more biomedcentral.com/submissions 